\title{
Pressure-induced phase transitions and superconductivity in a black phosphorus single crystal
}

\author{
Xiang Li \\ Beijing Institute of Technology, Beijing, China, People's Republic of; \\ xiangli@bit.edu.cn
}

\begin{abstract}
A high-pressure study of a black phosphorus crystal leads to a rich phase diagram, including a Lifshitz-type semiconductor-semimetal transition, a Weyl semimetal, and superconductivity as well as structural phase transitions. Transport properties and quantum oscillations under high pressure provide critically valuable information to understand the physics behind these new phases. These properties have been measured reliably under hydrostatic pressure and magnetic field with a large-volume apparatus. Superconductivity in the A7 phase has been found to exhibit the largest magnetoresistance effect in its normal state so far. The BCS superconductivity in the A7 phase as identified by the experiment can be accounted for by a first-principles calculation.
\end{abstract}

Keywords: high pressure, black phosphorus, phase transition, topological property 